\title{
Optically-pumped InAs/GaAs quantum-dot microdisk lasers monolithically grown on on-axis $\mathrm{Si}$ (001) substrate
}

Zhou, Taojie, Tang, Mingchu, Xiang, Guohong, Chen, Siming, Liu, Huiyun, et al.

Taojie Zhou, Mingchu Tang, Guohong Xiang, Siming Chen, Huiyun Liu, Zhaoyu Zhang, "Optically-pumped InAs/GaAs quantum-dot microdisk lasers monolithically grown on on-axis Si (001) substrate," Proc. SPIE 11182, Semiconductor Lasers and Applications IX, 1118208 (19 November 2019); doi: $10.1117 / 12.2538773$

SPIE. Event: SPIE/COS Photonics Asia, 2019, Hangzhou, China 


\title{
Optically-pumped InAs/GaAs quantum-dot microdisk lasers monolithically grown on on-axis $\mathrm{Si}(001)$ substrate
}

\author{
Taojie Zhou ${ }^{1, \dagger}$, Mingchu Tang ${ }^{2, \dagger}$, Guohong Xiang ${ }^{1}$, Siming Chen ${ }^{2}$, Huiyun Liu ${ }^{2}$, and Zhaoyu \\ Zhang ${ }^{1}$ \\ ${ }^{1}$ School of Science and Engineering, The Chinese University of Hong Kong, Shenzhen, \\ Guangdong, 518172, P.R. China \\ ${ }^{2}$ Department of Electronic and Electrical Engineering, University College London, London, \\ WC1E 7JE,UK \\ ${ }^{\dagger}$ T.Zhou and M.Tang contributed equally
}

\begin{abstract}
Monolithic integration of III-V semiconductor materials on planar on-axis silicon (001) is one of the most promising method for low-cost and scalable photonic integrated circuits. Here, we present InAs/GaAs quantum dots microdisk lasers monolithically grown on on-axis Si (001) substrate with ultra-low lasing threshold under room-temperature continues-wave optical pumping. The promising lasing characteristics of the microdisk lasers with ultra-low threshold and small footprint represent a major advance towards large-scale, low-cost integration of laser sources on silicon-based platform.
\end{abstract}

Keywords: Microdisk laser, ultra-low threshold, photonic integrated circuits

\section{INTRODUCTION}

Advanced silicon photonics have emerged as a promising candidate for next-generation chip-scale data communication networks and data centers. ${ }^{1,2}$ Monolithic growth of III-V semiconductor materials on silicon is one of the most promising route toward low-cost and scalable photonic integration, ${ }^{3}$ which has been proven to be difficult due to the conjunction of large thermal-, lattice-, and polarity- mismatches between Si substrate and III-V layer. High-performance quantum dot (QD) or quantum well (QW) lasers epitaxially grown on silicon has been demonstrated by using off-cut $\left(4^{0}-6^{0}\right)$ Si substrates, ${ }^{4}$ patterned on-axis $\mathrm{Si}(001),{ }^{5}$ Ge-on- $\mathrm{Si}^{6}$ or $\mathrm{GaP} / \mathrm{Si}$ (001) substrates. ${ }^{7}$

Compared with ridge waveguide lasers or DFB lasers monolithically grown on $\mathrm{Si}$, the whispering gallery modes (WGMs) microdisk lasers with small footprint configurations and ultra-low threshold allow for incorporating compact and efficient laser sources on a CMOS compatible platform. ${ }^{8}$ However, room-temperature CW pumped microdisks QDs lasers with ultra-low lasing threshold directly grown on planar on-axis $\mathrm{Si}(001)$ substrate has not been demonstrated.

In this work, we demonstrate low-threshold lasing in InAs/GaAs QDs microdisk lasers monolithically grown on on-axis $\mathrm{Si}(001)$ substrate under room-temperature $\mathrm{CW}$ optical pumping. ${ }^{9}$ Lasing emission of microdisk lasers with diameter $(D) \sim 1.9 \mu \mathrm{m}$ and sub-wavelength scale $(D \sim 1.1 \mu \mathrm{m})$ were illustrated, and ultra-low threshold $\sim 3 \mu \mathrm{W}$ was obtained.

\section{MATERIAL GROWTH AND FABRICATION}

The InAs/GaAs QDs microdisk lasers were monolithically grown on planar on-axis $\mathrm{Si}(001)$ substrates without any intermediate buffer layer. ${ }^{10}$ To overcome the antiphase boundaries (APBs) problem, a $400 \mathrm{~nm}$ APBs free

Send correspondence to Siming Chen, Huiyun Liu and Zhaoyu Zhang

Siming Chen: E-mail: siming.chen@ucl.ac.uk

Huiyun Liu: E-mail: huiyun.liu@ucl.ac.uk

Zhaoyu Zhang: E-mail: zhangzy@cuhk.edu.cn 

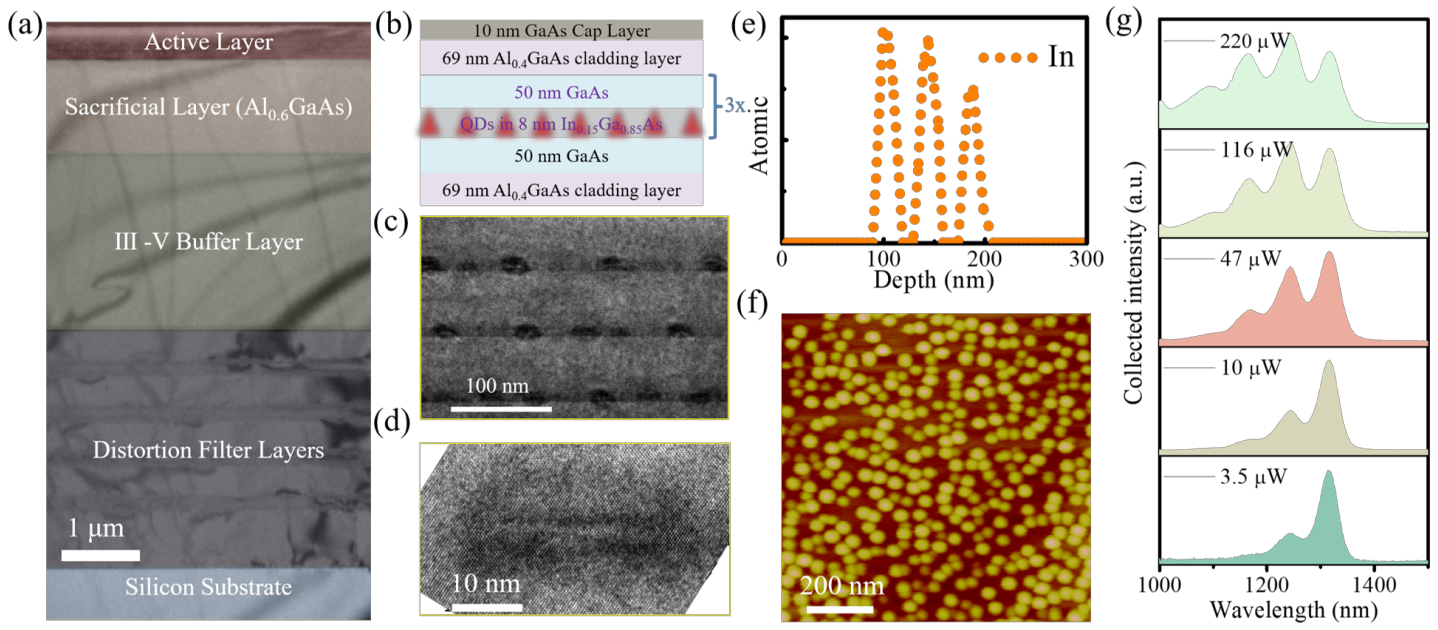

Figure 1. (a) TEM image of the microdisk structure grown on planar on-axis Si (001) substrate. (b) Schematic illustration of the epitaxial structure of microdisk region. (c) and (d) show the high-resolution TEM image of the three-stack InAs QDs layers and a single QD, respectively. (e) Atomic distribution profile of In obtained by XPS. (f) AFM image of uncapped InAs/GaAs QDs grown on $\mathrm{Si}(001)$ substrate. (g) Room temperature PL spectra of the as grown structure at various input power.

epitaxial GaAs film was first deposited on on-axis $\operatorname{Si}(001)$ substrate. Figure .1(a) shows the cross-sectional transmission electron microscope (TEM) image of the microdisk structure grown on planar on-axis Si (001) substrate. The epitaxial structure of microdisk region is schematically demonstrated in Figure .1(b). The microdisk region consists of three-layer well-developed $\mathrm{InAs} / \mathrm{In}_{0.15} \mathrm{Ga}_{0.85} \mathrm{As} / \mathrm{GaAs}$ dot-in-well (DWELL) active layer separated by $50 \mathrm{~nm}$ GaAs spacers, and sandwiched by two symmetrical $69 \mathrm{~nm}$ thick $\mathrm{Al}_{0.4} \mathrm{GaAs}_{\mathrm{A}}$ cladding layers with $10 \mathrm{~nm}$ cap layer. Figures .1(c) and (d) illustrate respectively the high-resolution TEM image of the grown three-stack InAs/GaAs QDs layers and a single QD. Figure .1(e) shows the atomic distribution profile of In as a function of depth from the surface on epitaxial chip obtained by X-ray photoelectron spectroscopy (XPS). The QDs monolithically grown on Si present good uniformity with density $\sim 4 \times 10^{10} \mathrm{~cm}^{-2}$ with typical size $\sim 25$ $\mathrm{nm}$ in diameter and $\sim 8 \mathrm{~nm}$ in height determined from the AFM and TEM images. Figure. 1(g) demonstrates the room temperature photoluminescene (PL) spectra of the as grown structure at various input power from 2.5 $\mu \mathrm{W}$ to $220 \mu \mathrm{W}$, indicating ground state emission was at $\sim 1.3 \mu \mathrm{m}$ telecommunication wavelength band. And higher excited states were observed as increasing the pumping power.

The microdisks pattern are defined by using electron beam lithography (EBL) with ZEP520A electron beam resist. Silicon dioxide with thickness around $120 \mathrm{~nm}\left(\mathrm{SiO}_{2}\right)$ is deposited on the wafer by Plasma Enhanced Chemical Vapor Deposition (PECVD) and used as the hard mask. The microdisk patterns are transferred from the resist into the hard mask by using reactive ion etching (RIE). After removing the resist ZEP520A, the hard mask patterns are further transferred through the active region (thickness $\sim 360 \mathrm{~nm}$ ) by using an inductively coupled plasma RIE (ICP-RIE). Then wet-etching is used to remove the $\mathrm{SiO}_{2}$ hard mask and form the supporting pedestal. The fabricated microdisks were characterized in a micro-PL ( $\mu$-PL) system at room-temperature and CW optically pumped by using $632.8 \mathrm{~nm}$ He-Ne laser with focus spot size $\sim 3 \mu \mathrm{m}$.

\section{RESULTS AND DISCUSSION}

To obtain a single mode lasing emission, one way is decreasing the diameter of microdisk cavity due to the free spectral range (FSR) becomes larger with smaller diameter of microdisk cavity and well-separated resonant peaks. Here, the lasing characteristics of a sub-wavelength microdisk lasers with $D \sim 1.1 \mu \mathrm{m}$ and $1.4 \mu \mathrm{m}$ were measured. Figure .2(a) presents the lasing spectra of the microdisk with $D \sim 1.1 \mu \mathrm{m}$ and $1.4 \mu \mathrm{m}$. A large FSR $\sim 159 \mathrm{~nm}(\sim 116 \mathrm{~nm})$ for microdisk laser with $D \sim 1.1 \mu \mathrm{m}(1.4 \mu \mathrm{m})$ of the same radial order was observed. The measured FSR is much larger than the linewidth $(\sim 46 \mathrm{~nm})$ of the ground state emission, which substantially can support one resonate frequency of first-order WGMs within the ground state. In addition, the 

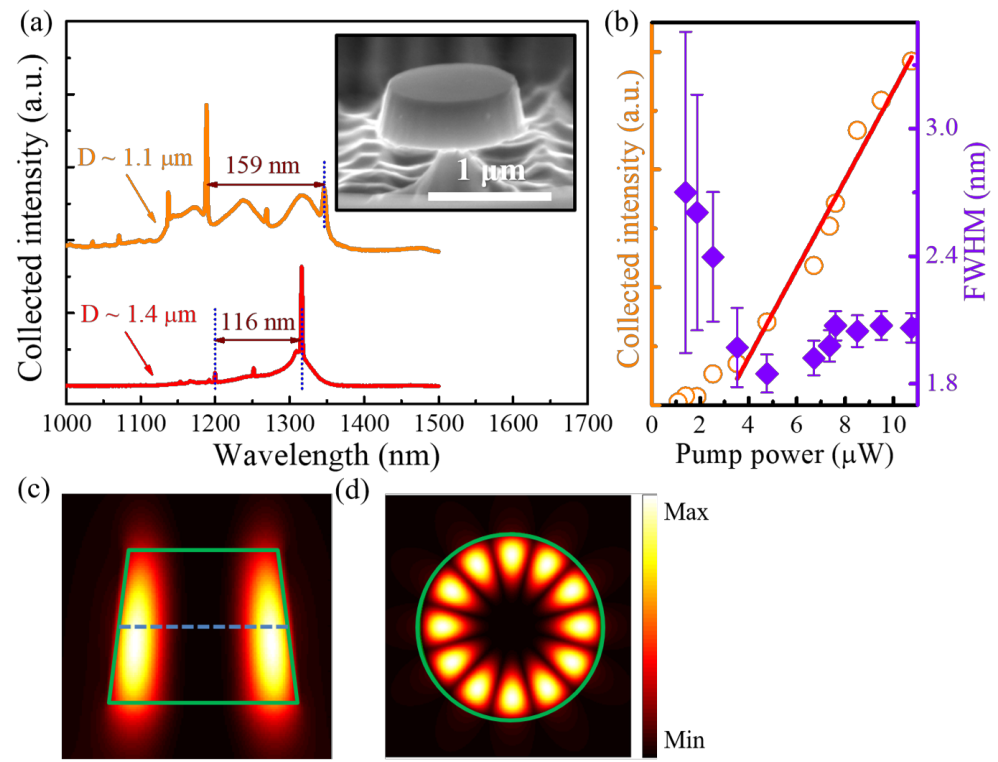

Figure 2. Measured lasing spectra of the microdisk laser with $D \sim 1.1 \mu \mathrm{m}$ and $\sim 1.4 \mu \mathrm{m}$. The inset SEM image shows the fabricated sub-wavelength scale microdisk laser. (b) L-L curve and FWHM of lasing peak $\sim 1189 \mathrm{~nm}$ of the subwavelength scale microdisk laser with $D \sim 1.1 \mu \mathrm{m}$, showing the lasing threshold of $\sim(2.9 \pm 0.4) \mu \mathrm{W}$. (c) and (d) The calculated cross-section and top-view of the magnetic field profiles for the $T E_{1,6}$ mode, respectively. The green line represents the boundary of the microdisk and the blue dashed line demonstrates the central plane of the microdisk.

mode positions (cavity resonate frequencies) are highly dependent on the structural parameters of the cavity. During the optical pumping process, the lasing emission peak from the excited states at $1189 \mathrm{~nm}$ (ground state at $1315 \mathrm{~nm})$ dominates the lasing spectra of the microdisk laser with $D \sim 1.1 \mu \mathrm{m}(\sim 1.4 \mu \mathrm{m})$. A lasing peak with weaker intensity of the ground state is also observed for the microdisk laser with $D \sim 1.1 \mu \mathrm{m}$, for which there is not clear mode switching from the ground state to the excited states with gradually increasing the pumping power. The lasing in the excited states is due mainly to the mode selection of the sub-wavelength scale microdisk cavity. The inset in Figure .2(a) shows the SEM image of a fabricated sub-wavelength micordisk. The collected intensity and full width at half maximum (FWHM) of lasing peak at $\sim 1189 \mathrm{~nm}$ was demonstrated in Figure .2 (b), indicating the lasing threshold $\sim(2.9 \pm 0.4) \mu \mathrm{W}$. The mode profile of the lasing peak at $1189 \mathrm{~nm}$ was calculated by using 3D finite-difference time-domain (3D-FDTD) method, which was identified as $T E_{1,6}$ mode. Figures $.2(\mathrm{c})$ and $2(\mathrm{~d})$ show respectively the cross-section and top-view of calculated magnetic field profiles for $T E_{1,6}$, of which the green line indicates the boundary of microdisk resonator and the blue dash line demonstrates the central plane of microdisk. As indicated form Figure .2(c), the center plane of WGMs was not completely overlapped with the emitter, which may cause the broad background emission of lasing spectra.

Besides, microdisk lasers with four layers of QDs were studied to compare the optical property of microdisk lasers with three layers of QDs. Figure. 3(a) shows the corresponding high-resolution TEM image of the active region for the microdisk with four-stack InAs/GaAs QDs layers, the inset illustrates a high-resolution TEM image of a single QD. Figure. 3(b) presents the lasing spectra of a microdisk laser $(D \sim 1.9 \mu \mathrm{m})$. The cold cavity quality-factor ( $Q$-factor) at $1336 \mathrm{~nm}$ was estimated $\sim 1349\left(Q=\frac{\lambda}{\Delta \lambda}, \Delta \lambda\right.$ was mode linewidth extracted at a pump power below the lasing threshold). The inset in Figure. 3(b) shows the schematic diagram of layer structure with four layers of QDs. Figrue .3(c) illustrates the corresponding collected intensity and FWHM of the lasing peak at $\sim 1336 \mathrm{~nm}$ with lasing threshold $\sim(4.6 \pm 0.3) \mu \mathrm{W}$. The lasing threshold was slightly higher than microdisk laser with the same size composed of three layers of QDs,${ }^{9}$ but is almost in a same order of magnitude. 

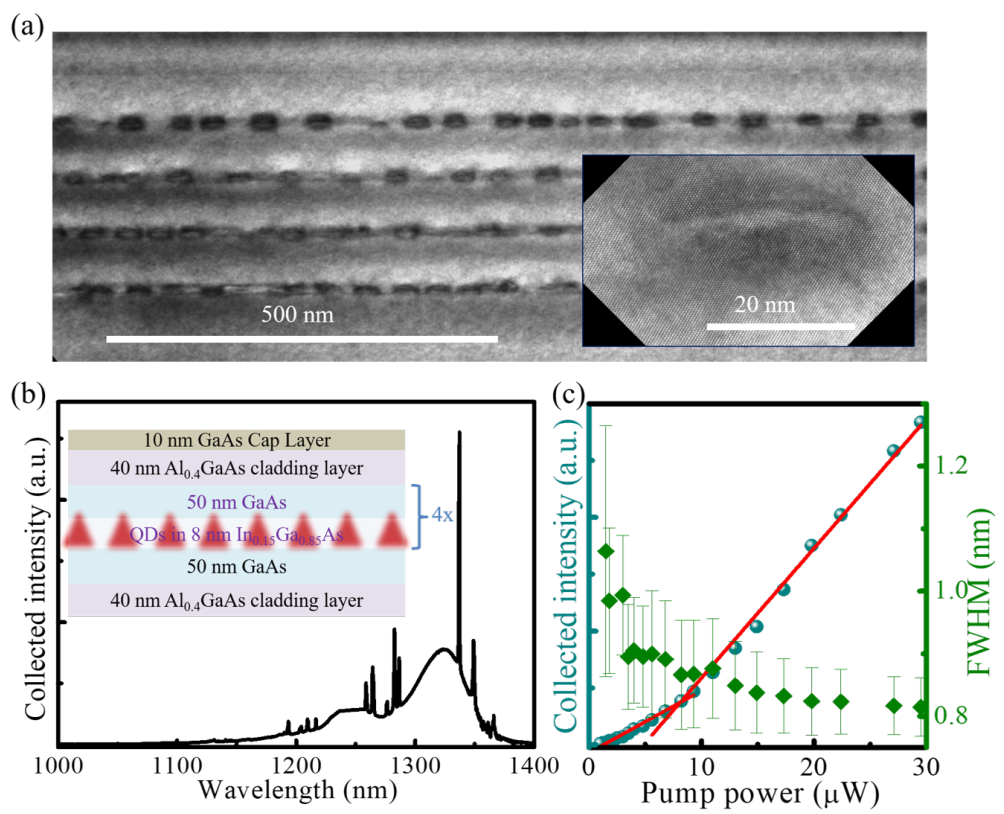

Figure 3. (a) High-resolution TEM image of the four-stack InAs QDs layers with the inset presenting a single QD. (b) Measured spectra above lasing threshold, the inset shows the layer structure of microdisk active region with four layers of QDs grown on planar on-axis Si (001) substrate. (c) Corresponding collected intensity and FWHM of the lasing peak at $\sim 1336 \mathrm{~nm}$, showing the lasing threshold $\sim(4.6 \pm 0.3) \mu \mathrm{W}$.

\section{CONCLUSION}

In conclusion, we present InAs/GaAs QDs microdisk lasers monolithically grown on planar on-axis Si (001) substrate. The microdisk lasers were CW optically pumped at room temperature, of which ultra-low lasing threshold $\sim 3 \mu \mathrm{W}$ was obtained. The promising lasing characteristics of the microdisk lasers with ultra-low threshold and small footprint monolithic grown on Si (001) substrate provide a viable route towards the largescale, low-cost integration of laser sources on a silicon platform.

\section{ACKNOWLEDGMENTS}

This work were supported by Shenzhen Key Laboratory Project (ZDSYS201603311644527); Shenzhen Fundamental Research Fund (JCYJ20150611092848134, JCYJ20150929170644623); Shenzhen Science and Technology Innovation Fund (KQCX20140522143114399); President's Fund (PF01000154); UK EPSRC (EP/P000886/1, EP/P006973/1); EPSRC National Epitaxy Facility;European project H2020-ICT-PICTURE (780930) and Royal Academy of Engineering (RF201617/16/28).

\section{REFERENCES}

[1] Liang, D. and Bowers, J. E., "Recent progress in lasers on silicon," Nature Photonics 4(8), 511-517 (2010).

[2] Thomson, D., Zilkie, A., Bowers, J. E., Komljenovic, T., Reed, G. T., Vivien, L., Marrismorini, D., Cassan, E., Virot, L., and Fédéli, J. M., "Roadmap on silicon photonics," Journal of Optics 18(7), 073003 (2016).

[3] Chen, R., Ng, K. W., Ko, W. S., Parekh, D., Lu, F., Tran, T.-T. D., Li, K., and Chang-Hasnain, C., "Nanophotonic integrated circuits from nanoresonators grown on silicon," Nature Communications 5, 4325 (2014).

[4] Chen, S., Li, W., Wu, J., Jiang, Q., Tang, M., Shutts, S., Elliott, S. N., Sobiesierski, A., Seeds, A. J., Ross, I., et al., "Electrically pumped continuous-wave III-V quantum dot lasers on silicon," Nature Photonics 10(5), 307 (2016). 
[5] Wan, Y., Li, Q., Liu, A. Y., Chow, W. W., Gossard, A. C., Bowers, J. E., Hu, E. L., and Lau, K. M., "Subwavelength InAs quantum dot micro-disk lasers epitaxially grown on exact Si (001) substrates," Applied Physics Letters 108(22), 221101 (2016).

[6] Liu, H., Wang, T., Jiang, Q., Hogg, R., Tutu, F., Pozzi, F., and Seeds, A., "Long-wavelength InAs/GaAs quantum-dot laser diode monolithically grown on Ge substrate," Nature Photonics 5(7), 416 (2011).

[7] Jung, D., Norman, J., Kennedy, M. J., Shang, C., Shin, B., Wan, Y., Gossard, A. C., and Bowers, J. E., "High efficiency low threshold current $1.3 \mu \mathrm{m}$ InAs quantum dot lasers on on-axis (001) GaP/Si," Applied Physics Letters 111(12), 122107 (2017).

[8] Shi, B., Zhu, S., Li, Q., Wan, Y., Hu, E. L., and Lau, K. M., "Continuous-wave optically pumped $1.55 \mu \mathrm{m}$ InAs/InAlGaAs quantum dot microdisk lasers epitaxially grown on silicon," ACS Photonics 4(2), 204-210 (2017).

[9] Zhou, T., Tang, M., Xiang, G., Fang, X., Liu, X., Xiang, B., Hark, S., Martin, M., Touraton, M.-L., Baron, T., et al., "Ultra-low threshold InAs/GaAs quantum dot microdisk lasers on planar on-axis Si (001) substrates," Optica 6(4), 430-435 (2019).

[10] Alcotte, R., Martin, M., Moeyaert, J., Cipro, R., David, S., Bassani, F., Ducroquet, F., Bogumilowicz, Y., Sanchez, E., and Ye, Z., "Epitaxial growth of antiphase boundary free GaAs layer on $300 \mathrm{~mm} \operatorname{Si}(001)$ substrate by metalorganic chemical vapour deposition with high mobility," Apl Materials 4(4), 083514 (2016). 